Invited commentary

Paediatrics Today 2016;12(1):191-194

DOI $10.5457 /$ p2005-114.159

\title{
WHAT DID THE ELEVENTH WORLD KIDNEY DAY TELL US?
}

\author{
Amira PECO-ANTIĆ \\ University Children's Hospital, Belgrade, Serbia \\ Correspondence: \\ amirapecoantic@yahoo.com \\ Tel.: + 38111603289191
}

Received /Accepted: 20 June 2016

Key words: Acute kidney injury - Chronic kidney disease - Renoprotection - Children.

The Eleventh World Kidney Day (WKD) was marked on March 10, 2016 around the world. This annual event, sponsored jointly by the International Society of Nephrology (ISN) and the International Federation Foundation for Kidney Disease (IFKF), has become a very important way for the general public and the world's policy makers to learn about the importance of kidney disease and its consequences. This year's WKD was dedicated to children's kidney diseases, which may go beyond childhood and become adult kidney disease with possible complications for other organs (1). The main motto of the 11th WKD was: "Act early to prevent it!" To achieve this very ambitious target, it is essential to lead a healthy life style from early childhood, and continuing through the whole life, to detect preventable kidney damage in time, and to treat children with inborn and acquired disorders of the kidney.

Acute and chronic kidney disease in childhood differ in comparison with those in adults. Also, significant differences exist within the pediatric population depending on age, and ethnic and regional affiliations, which are closely related to genetic and socioeconomic factors.

\section{Acute kidney injury}

The frequency of acute kidney injury (AKI) in childhood is highest in the first month of life, and then decreases progressively (2). The predominant causes of pediatric AKI vary in different regions of the world (3). The common occurrence of infections and gastroenteritis, leading to severe dehydration and subsequent acute tubular necrosis are the main factors for AKI in rural developing-country setting. By contrast, the complications of very sophisticated medical treatment, such as cardiac surgery, chemotherapy, and organ and bone marrow transplant, have become more prevalent AKI causes in tertiary care units in developed countries in recent years (4-6). The mortality rate in children with AKI is considered to depend largely on the nature of the underlying disease and the availability of resources to treat AKI. Mortality is usually very high (up to 90\%) in patients with AKI related to multi-organ failure, while survivors often suffer from irreversible complications (7). In tertiary-intensive care pediatric unit patients, $\sim 10 \%$ develop chronic kidney disease (CKD) 1-3 years after AKI (8). To improve the outcome of AKI in general, early diagnosis is essential, which will likely achieved by the use of new biomarkers, such as neutrophil gelatinase-associated lipocalin, kidney injury molecule-1, interleukin 18 and liver-type fatty acid binding protein (9). To 
address the problem of AKI treatment in the developing nations of sub-Saharan Africa and South East Asia, the ISN initiated the Saving Young Lives Project, which aims both to prevent AKI with prompt treatment of infection and/or delivery of appropriate fluid and electrolyte therapy, and to treat AKI when it occurs, including the provision of acute peritoneal dialysis (1). It is expected that the Project activities will ensure by 2025 that nobody will die from preventable and acute kidney injury (1).

\section{Chronic kidney disease}

The existing epidemiological data on CKD in children mainly focus on renal replacement therapy (RRT), which covers only part of the population suffering from CKD during childhood, as a considerable number of children with renal impairment will reach endstage renal disease (ESRD) in adulthood. The prevalence of ESRD in childhood has been variously reported as 15 to 74.7 per million children (10). It constitutes only a few percent of the total ESRD patient population (11). In contrast to adult CKD, which is predominately caused by diabetic nephropathy, hypertension, and autosomal dominant polycystic kidney disease, the main causes of pediatric CKD are congenital anomalies of the kidney and urinary tract (CAKUT) at approximately $50 \%$, followed by hereditary nephropathies and glomerulonephritis $(10,11)$. Unlike in adults, CKD in children is manifested by growth and development disorders. Further, the life expectancy of children with $\mathrm{CKD}$ is longer than in adult patients. Therefore, more intensive therapy is required to slow the progression of CKD. The last decade has witnessed enormous achievements in this field. In addition to standard renoprotective therapy that works by normalization of blood pressure, proteinuria, hyperphos- phataemia, anemia and hyperuricemia, new drugs offer great hope (Table 1) (12).

According to the ESCAPE trial data, strict blood pressure control ( $\sim$ 5othpercentile) retards progression of $\mathrm{CKD}$ in children, irrespective of the type of underlying kidney disease (13). Vitamin D may also promote renoprotection by suppressing renin transcription through cross-talk between RAAS and vitamin D-fibroblast growth factor-23 (FGF-23)-Klotho pathways (14). In line with the latest findings on the role of inflammation in the progression of CKD, therapeutic blockade of leucocytes chemokine receptors inhibits secretion of the proinflammatory, proapoptotic and profibrotic cytokines that perpetuate renal tissue destruction (12). The role of the gut as a major source of inflammation adds also a novel therapeutic tool, healthy nutrition, to delay CKD progression.

Very recently, progress in drug development in line with advances in genetics, have given hope for some kidney diseases including atypical HUS, (with the advent of a monoclonal antibody that specifically blocks C5 activation) (15), polycystic kidney disease (vasopressin receptor antagonists retard cyst growth and preserve kidney function) (16), and primary and secondary amyloidosis (IgG anti- Serum amyloid protein antibodies clears tissue amyloid deposits) (12). However, the benefit from modern therapy is not available to all children who need it because of the high cost of the drugs. Even RRT is not available to all children with ESRD. In developing countries, dialysis is often not offered, while undeveloped cadaveric transplantation might lead to trade in paid organ donation. The WKD provides the ideal opportunity to detect and discuss these and many other problems related to kidney diseases, and to initiate comprehensive action to resolve them. 
Table 1 Therapeutic intervention for renoprotection

\begin{tabular}{|c|c|c|}
\hline Therapeutic modality & Mechanism of action & Primary end points \\
\hline \multicolumn{3}{|l|}{ Standard } \\
\hline RAS blockers (ACEI, ARB) & $\mathrm{BP} \downarrow$ & 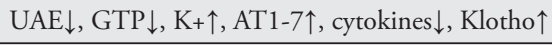 \\
\hline Aldosterone antagonists & $\mathrm{BP} \downarrow$ & $\mathrm{UAE} \downarrow, \mathrm{K}+\uparrow$ \\
\hline $\begin{array}{l}\text { Hypouricemic agents } \\
\text { Allpurinol } \\
\text { Febuxostst }\end{array}$ & Uric acid $\downarrow$ & Renal events $\downarrow$, CV events $\downarrow$, CKD progression $\downarrow$ \\
\hline Sodium bicarbonate & $\mathrm{HCO} 3-\uparrow$ & PH $\uparrow$, Ptn catabolism $\downarrow$, GFR decline $\downarrow$ \\
\hline $\begin{array}{l}\text { Phosphate binders } \\
\text { Calcium based } \\
\text { Sevelamer }\end{array}$ & $\mathrm{P} \downarrow$ & $\begin{array}{l}\mathrm{PTH} \downarrow, \mathrm{VC} \uparrow, \mathrm{P} \downarrow, \mathrm{PTH} \downarrow \text {, Stop VC, Mortality } \downarrow \text {, } \\
\text { Uric acid } \downarrow \text {, Cholesterol } \downarrow \text {, LDL } \downarrow \text {, inflammation } \downarrow \text {, } \\
\text { Cardiovascular events } \downarrow\end{array}$ \\
\hline \multicolumn{3}{|l|}{ Nuvel } \\
\hline $\begin{array}{l}\text { Chemokine ligand and } \\
\text { receptor antagonists }\end{array}$ & $\begin{array}{l}\text { Block CCR1 receptors on } \\
\text { leucocyte surface }\end{array}$ & $\mathrm{LI} \downarrow, \mathrm{IF} \downarrow, \mathrm{TI} \downarrow$, and improved \\
\hline Pentoxifylline & Anti-inflammatory & UAE $\downarrow$, eGFR loss $\downarrow$ \\
\hline VDRA & Improves GM sieving, antifibrotic & UAE $\downarrow$, eGFR loss $\downarrow$ \\
\hline $\begin{array}{l}\text { IAP, Mediterranean diet, } \\
\text { Bound phosphorus, Vitamin K }\end{array}$ & Restores intestinal microbiota & IAP $\uparrow$ eGFR loss $\downarrow[184]$ \\
\hline SOD mimetic, Tempol & Oxidative stress $\downarrow$ & $\mathrm{UAE} \downarrow, \mathrm{GS} \downarrow, \mathrm{TID} \downarrow$ \\
\hline SRA, Sarpogrelate & Antiplatelet & $\mathrm{UAE} \downarrow$ \\
\hline Monoclonal antibody-eculizumab & Blocks complement $\mathrm{C} 5$ activation & Terminal complement blockade \\
\hline V2RA, Tolvaptan & V2 receptor blocker & No. of cysts $\downarrow$, Growth of cysts $\downarrow$ \\
\hline IgG anti-SAP antibodies & Binds SAP within amyloid tissue & Clearance of tissue amyloid deposits \\
\hline
\end{tabular}

RAS=Renin angiotensin system; ACEI=angiotensin converting enzyme inhibitors; $A R B$ angiotensin receptor blockers; $\mathrm{BP}=\mathrm{Blood}$ pressure; $\mathrm{UAE}=\mathrm{Urine}$ albumin excretion; $\mathrm{GTP}=\mathrm{Glomerular}$ tuft pressure; $\mathrm{K}=$ Potassium; AT1-7=Angiotensin 1-7; $\mathrm{CV}=$ Cardiovascular; $\mathrm{CKD}=$ Chronic kidney disease; $\mathrm{HCO} 3=$ Bicarbonate; Ptn=Protein; GFR=Glomerular filtration rate; $\mathrm{P}=$ Phosphorus; PTH=Parathormone; VS=Vascular calcification; TG=Triglycerides; HDL=High density lipoproteins. LI=Leucocyte infiltration; IF=Interstitial fibrosis; TI=Tubular injury; KFTs=Kidney function tests; UAE=Urine albumin excretion; DN=Diabetic nephropathy; eGFR=Estimated glomerular filtration rate; VDRA=Vitamin D receptor agonists; GM=Glomerular membrane; IAP=Intestinal alkaline phosphatase; $\mathrm{SOD}=$ Superoxide dismutase; GS=Glomerulosclerosis; TID=Tubulointerstitial disease; $\mathrm{SRA}=$ Serotonin receptor antagonist; V2RA=Vasopressin receptor antagonist; SAP=Serum amyloid protein.

\section{Conclusion}

The Eleventh WKD is a global alertness and education campaign that highlights renal disease and children. The general message is "a collaborative modality against the renal diseases in children that lead to endstage renal failure, through increasing community outreach, improved economic opportunity, better education, and access to preventive strategies for individuals at highest risk."

Conflict of interest: The author declares that she has no conflict of interest.

\section{References}

1. Ingelfinger R. J, Kalantar-Zadeh K, Schaefer F. World Kidney Day 2016. Averting the Legacy of Kidney Disease: Focus on Childhood. Paediatrics Today. 2016;12(1):115-23.

2. Hui-Stickle S, Brewer ED, Goldstein SL. Pediatric ARF epidemiology at a tertiary care center from 1999 to 2001. Am J Kidney Dis. 2005;45:96-101.

3. Goldstein SL. Overview of pediatric renal replacement therapy in acute renal failure. Artif organs 2003;27:781-5.

4. Santos CR, Branco PQ, Gaspar A, Bruges M, Anjos R, Gon-çalves MS, et al. Use of peritoneal dialysis after surgery for congenital heart disease in children. Perit Dial Int. 2012;32:273-9. 
5. Humphreys BD, Soiffer RJ, Magee CC. Renal failure associated with cancer and its treatment: an update. J Am Soc Nephrol. 2005;16:151-61.

6. Detaille T, Anslot C, de Cléty SC. Acute kidney injury in pediatric bone marrow patients. Acta Clin Belg. 2007;62(Suppl 2):401-4.

7. Goldstein S, Currier H, Graf C. Outcome in children receiving continuous venovenous hemofiltration. Pediatrics. 2001;107:1309-12.

8. Mammen C, Al Abbas A, Skippen P, Nadel H, Levine D, Collet JP, et al. Long-term risk of CKD in children surviving episodes of acute kidney injury in the intensive care unit: a prospective cohort study. Am J Kidney Dis. 2012;59(4):523-30.

9. Peco-Antić A, Paripović D. Renal hypertension and cardiovascular disorder in children with chronic kidney disease. Srp Arh Celok Lek. 2014;142(12):113-7.

10. Warady BA, Chadha V. Chronic kidney disease in children: the global perspective. Pediatr Nephrol. 2007;22:1999-2009.

11. Peco-Antic A, Bogdanovic R, Paripovic D, Paripovic A, Kocev N, Golubovic E, et al. Epidemiology of chronic kidney disease in children in Serbia. Ser- bian Pediatric Registry of Chronic Kidney Disease (SPRECKID). Nephrol Dial Transplant. 2012; 27(5):1978-84.

12. Sharaf El Din UA, Salem MM, Abdulazim DO. Stop chronic kidney disease progression: Time is approaching. World J Nephrol. 2016 May 6;5(3):258-73.

13. Wühl E, Trivelli A, Picca S, Litwin M, Peco-Antic A, Zurowska A, et al; ESCAPE Trial Group. Strict blood-pressure control and progression of renal failure in children. N Engl J Med. 2009;361:163950.

14. Shroff R, Aitkenhead H, Costa N, Trivelli A, Litwin $\mathrm{M}$, Picca $S$, et al; ESCAPE Trial Group. Normal 25-Hydroxyvitamin D Levels Are Associated with Less Proteinuria and Attenuate Renal Failure Progression in Children with CKD. J Am Soc Nephrol. 2016;27(1):314-22.

15. Verhave JC, Wetzels JF, van de Kar NC. Novel aspects of atypical haemolytic uraemic syndrome and the role of eculizumab. Nephrol Dial Transplant. 2014;29(Suppl 4): iv131-iv141

16. Torres VE. Vasopressin receptor antagonists, heart failure, and polycystic kidney disease. Annu Rev Med. 2015;66:195-210. 